\title{
A new class of reconstructible graphs from some neighbourhood conditions
}

\author{
Tetsuya Hosaka and Yonghuo XiAO
}

(Received April 10, 2013; Revised October 28, 2013)

\begin{abstract}
In this paper, we provide a new class of reconstructible finite graphs. We show the following theorem: Let $k$ be a positive integer number. Let $\Gamma$ be a finite graph with at least 3 vertices. Suppose that $\Gamma$ satisfies the following two conditions: (i) for any two distinct vertices $w, w^{\prime} \in V(\Gamma),\left[w, w^{\prime}\right] \in E(\Gamma) \Leftrightarrow N(w)-\left\{w^{\prime}\right\} \not N(s)$ for any vertex $s \in V(\Gamma)$; (ii) there exists a vertex $v \in V(\Gamma)$ of degree $k$ such that for any $k$-vertices $v_{1}, v_{2}, \ldots, v_{k} \in V(\Gamma)-\{v\}$, there exists a vertex $u \in V(\Gamma)$ such that $S t^{2}(u, \Gamma) \cap\left\{v, v_{1}, v_{2}, \ldots, v_{k}\right\}=\emptyset$, where $N(w)$ is the full subgraph of $\Gamma$ whose vertex set is $\{v \in V(\Gamma) \mid[w, v] \in E(\Gamma)\}$ and $S t^{2}(u, \Gamma)=\bigcup\{S t(w, \Gamma) \mid w \in V(S t(u, \Gamma))\}$. Then the graph $\Gamma$ is reconstructible. We also provide some applications and examples.
\end{abstract}

Key words: reconstructible graph, flag complex.

\section{Introduction}

A graph $\Gamma$ is an ordered pair $(V(\Gamma), E(\Gamma))$ consisting of a set $V(\Gamma)$ of vertices and a set $E(\Gamma)$ of edges. We use $[u, v]$ to denote a pair of vertices $u, v \in V(\Gamma)$, and write $[u, v] \in E(\Gamma)$ or $[u, v] \notin E(\Gamma)$ to indicate whether or not they form an edge in $\Gamma$. If $v \in V(\Gamma)$, the vertex-deleted subgraph of $\Gamma$, denoted by $\Gamma_{V-\{v\}}$ or $\Gamma-\{v\}$, is the graph obtained from $\Gamma$ by deleting the vertex $v$ and its incident edges. A graph $\Gamma$ is finite if both its vertex set $V(\Gamma)$ and edge set $E(\Gamma)$ are finite. A graph $\Gamma$ is simple if it has no loops or multiple edges.

Two graphs $\Gamma$ and $\Delta$ are isomorphic, written $\Gamma \cong \Delta$, if there are bijections $\theta: V(\Gamma) \rightarrow V(\Delta)$ and $\sigma: E(\Gamma) \rightarrow E(\Delta)$ such that $e=[u, v]$ if and only if $\sigma(e)=[\theta(u), \theta(v)]$.

In this paper, we consider only graphs which are finite, simple and undirected.

A graph $\Gamma$ is said to be reconstructible, if any graph $\Gamma^{\prime}$ with the following property $(*)$ is isomorphic to $\Gamma$. 
(*) There exists a bijection $f: V(\Gamma) \rightarrow V\left(\Gamma^{\prime}\right)$ such that the subgraphs $\Gamma-\{v\}$ and $\Gamma^{\prime}-\{f(v)\}$ are isomorphic for any $v \in V(\Gamma)$.

The following open problem is well-known as the Reconstruction Conjecture.

Problem (Reconstruction Conjecture) Is every finite simple graph with at least three vertices reconstructible?

Some classes of reconstructible graphs are known (cf. [2], [6], [7], [8], [9]) as follows: Let $\Gamma$ be a finite graph with at least three vertices.

(1) If $\Gamma$ is a regular graph, then it is reconstructible.

(2) If $\Gamma$ is a tree, then it is reconstructible.

(3) If $\Gamma$ is not connected, then it is reconstructible.

(4) If $\Gamma$ has at most 11 vertices, then it is reconstructible.

Now we provide some definitions.

Definition 1.1 (cf. [1]) Let $\Gamma$ be a finite graph and let $v$ be a vertex of the graph $\Gamma$. Then the degree of the vertex $v$, denoted by $\operatorname{deg}(v)$, is the number of edges with $v$ as an end vertex in $\Gamma$. The neighbourhood graph of the vertex $v$, denoted by $N(v)$, is the full subgraph of $\Gamma$ whose vertex set is $\{w \in V(\Gamma) \mid[w, v] \in E(\Gamma)\}$. The star graph of the vertex $v$, denoted by $S t(v, \Gamma)$, is the full subgraph of $\Gamma$ on the vertex subset $\{v\} \cup V(N(v))$.

The purpose of this paper is to prove the following main theorem, which provide a new class of reconstructible graphs. We prove this theorem in Section 2.

Theorem 1.2 Let $k$ be a positive integer number. Let $\Gamma$ be a finite graph with at least 3 vertices. Suppose that $\Gamma$ satisfies the following two conditions:

(i) for any two distinct vertices $w, w^{\prime} \in V(\Gamma)$,

$$
\left[w, w^{\prime}\right] \in E(\Gamma) \Leftrightarrow N(w)-\left\{w^{\prime}\right\} \not N(s) \text { for any vertex } s \in V(\Gamma) ;
$$

(ii) there exists a vertex $v \in V(\Gamma)$ of degree $k$ such that for any $k$-vertices $v_{1}, v_{2}, \ldots, v_{k} \in V(\Gamma)-\{v\}$, there exists a vertex $u \in V(\Gamma)$ such that

$$
S t^{2}(u, \Gamma) \cap\left\{v, v_{1}, v_{2}, \ldots, v_{k}\right\}=\emptyset,
$$




$$
\text { where } S t^{2}(u, \Gamma)=\bigcup\{S t(w, \Gamma) \mid w \in V(S t(u, \Gamma))\} \text {. }
$$

Then the graph $\Gamma$ is reconstructible.

Remark The right-to-left implication in the condition (i) holds for any graph, that is, in any graph $\Gamma$, the following condition holds:

( $\hat{\mathrm{i}})$ for any two distinct vertices $w, w^{\prime} \in V(\Gamma)$,

$$
\left[w, w^{\prime}\right] \in E(\Gamma) \Leftarrow N(w)-\left\{w^{\prime}\right\} \approx N(s) \text { for any vertex } s \in V(\Gamma) .
$$

Indeed, if $\left[w, w^{\prime}\right] \notin E(\Gamma)$, then $N(w)-\left\{w^{\prime}\right\} \cong N(w)$. Hence $N(w) \approx$ $N(s)$ for any vertex $s \in V(\Gamma)$. It is a contradiction.

Thus, the converse left-to-right implication is essential in the condition (i).

We can easily construct graphs satisfying the condition (ii) in Theorem 1.2. Indeed, if a graph has enough many vertices and every vertex has small degree, then the condition (ii) holds. We also note that the condition (ii) is a key condition and we essentially use this condition in the proof of Theorem 1.2 .

We introduce some examples and applications of Theorem 1.2 in Section 3. We need some defitions.

Definition 1.3 (cf. [5]) A simplicial complex $L$ is called a flag complex, if the following condition holds:

$(* *)$ For any vertex set $\left\{s_{0}, \ldots, s_{n}\right\}$ of $L$, if $\left\{s_{i}, s_{j}\right\}$ spans 1 -simplex in $L$ for any $i, j \in\{0, \ldots, n\}$ with $i \neq j$, then the vertex set $\left\{s_{0}, \ldots, s_{n}\right\}$ spans $n$-simplex in $L$.

Definition 1.4 (cf. [10]) Let $v$ be a vertex of a simplicial complex $L$. Then the open star of $v$ in $L$, denoted by $S^{\circ} t(v, L)$, is the union of the interiors of the simplices of $L$ that have $v$ as a vertex. Its closure, denoted by $S t(v, L)$, is called the closed star of $v$ in $L$. It is the union of all simplices of $L$ having $v$ as a vertex. The set $S t(v, L)-S t(v, L)$ is called the link of $v$ in $L$ and is denoted by $L k(v, L)$.

A finite graph $\Gamma$ uniquely determines a finite simplicial flag complex $L$ whose 1 -skeleton $L^{(1)}$ coincide with $\Gamma$ (cf. [5]). Then by Definition 1.1 and Definition 1.4, we have the following. 
(1) The neighbourhood graph $N(v)$ of the vertex $v$ in the graph $\Gamma$ coincide with the 1-skeleton of the link $L k(v, L)$ of the vertex $v$ in the simplicial flag complex $L$, i.e., $N(v)=L k(v, L)^{(1)}$.

(2) The star graph $S t(v, \Gamma)$ of the vertex $v$ in the graph $\Gamma$ coincide with the 1-skeleton of the closed star $S t(v, L)$ of the vertex $v$ in the simplicial flag complex $L$, i.e., $S t(v, \Gamma)=S t(v, L)^{(1)}$.

(3) $S t^{2}(v, \Gamma)=S t^{2}(v, L)^{(1)}$.

By these correspondence, we obtain the following corollary from Theorem 1.2.

Corollary 1.5 Let $k$ be a positive integer number and $i_{0}$ be a nonnegative number. Let $\Gamma$ be a finite graph with at least 3 vertices. Suppose that $\Gamma$ is the 1-skeleton of some simplicial flag complex $L$ which satisfies the following two conditions:

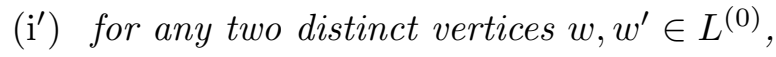

$$
\begin{aligned}
{\left[w, w^{\prime}\right] \in L^{(1)} \Leftrightarrow \widetilde{H}_{i_{0}}\left(L k(w, L)-\left\{w^{\prime}\right\}\right) } & \neq \widetilde{H}_{i_{0}}(L k(s, L)) \\
& \quad \text { for any vertex } s \in L^{(0)}
\end{aligned}
$$

(ii') there exists a vertex $v \in L^{(0)}$ of degree $k$ such that for any $k$-vertices $v_{1}, v_{2}, \ldots, v_{k} \in L^{(0)}-\{v\}$, there exists a vertex $u \in L^{(0)}$ such that

$$
S t^{2}(u, L) \cap\left\{v, v_{1}, v_{2}, \ldots, v_{k}\right\}=\emptyset,
$$

where $S t^{2}(u, L)=\bigcup\left\{S t(w, L) \mid w \in S t(u, L)^{(0)}\right\}$.

Then the graph $\Gamma$ is reconstructible.

Indeed, the condition ( $\left.\mathrm{i}^{\prime}\right)$ in Corollary 1.5 implies the condition (i) in Theorem 1.2.

Example 1.6 If $L$ is a simplicial flag complex and a homology $n$-manifold $(n \geq 1)$, then the condition ( $\left.\mathrm{i}^{\prime}\right)$ in Corollary 1.5 holds, where $i_{0}=n-1$. Detail of homology manifolds is found in [3, Section 5], [4], [10, p. 374], [11]. This is one of our motivations in this paper.

From Corollary 1.5, we also obtain the following.

Corollary 1.7 Let $L$ be a $(n+1)$-dimensional simplicial flag complex 
which is a triangulation of $L_{1} *\left\{a_{1}, a_{2}, \ldots, a_{k}\right\}$, where $L_{1}$ is a n-manifold and $k \geq 4$. If $L$ has enough vertices to satisfy the condition (ii') in Corollary 1.5 , then the graph $\Gamma=L^{(1)}$ is reconstructible.

We also provide some examples and remarks in Section 3.

\section{Proof of Theorem 1.2}

We prove Theorem 1.2.

Proof of Theorem 1.2. Let $\Gamma$ be a finite graph satisfing the two conditions (i) and (ii) in Theorem 1.2.

Then we show that the graph $\Gamma$ is reconstructible.

Let $\Gamma^{\prime}$ be a finite graph and let $V, E$ and $V^{\prime}, E^{\prime}$ be the vertex sets and the edge sets of the graphs $\Gamma$ and $\Gamma^{\prime}$ respectively.

Now we suppose that the condition $(*)$ in the introduction is satisfied.

To show that the graph $\Gamma$ is reconstructible, we prove that the two graphs $\Gamma$ and $\Gamma^{\prime}$ are isomorphic.

By the condition (ii), there exists a vertex $v \in V$ of degree $k$ such that for any $k$-vertices $v_{1}, v_{2}, \ldots, v_{k} \in V-\{v\}$, there exists a vertex $u \in V$ such that $S t^{2}(u, \Gamma) \cap\left\{v, v_{1}, v_{2}, \ldots, v_{k}\right\}=\emptyset$. We use the condition $(*)$ for the vertex $v \in V$. Then we see that $\Gamma-\{v\}$ and $\Gamma^{\prime}-\{f(v)\}$ are isomorphic, i.e, there exists an isomorphism $\varphi: \Gamma-\{v\} \rightarrow \Gamma^{\prime}-\{f(v)\}$. Let $v^{\prime}=f(v)$. Here it is well-known that the vertex $v$ in $\Gamma$ and the vertex $v^{\prime}$ in $\Gamma^{\prime}$ have the same degrees (cf. [9]). Since $v$ and $v^{\prime}$ have the same degrees,

$$
\left|V\left(\varphi^{-1}\left(N\left(v^{\prime}\right)\right)\right)\right|=\left|V\left(N\left(v^{\prime}\right)\right)\right|=\operatorname{deg}\left(v^{\prime}\right)=\operatorname{deg}(v)=k .
$$

By the condition (ii), there exists a vertex $a \in V$ such that

$$
S t^{2}(a, \Gamma) \cap\left(\{v\} \cup V\left(\varphi^{-1}\left(N\left(v^{\prime}\right)\right)\right)\right)=\emptyset .
$$

Then,

$$
\varphi\left(S t^{2}(a, \Gamma)\right) \cap\left(\left\{v^{\prime}\right\} \cup N\left(v^{\prime}\right)\right)=\emptyset,
$$

hence,

$$
\left.S t^{2}\left(\varphi(a), \Gamma^{\prime}\right)\right) \cap S t\left(v^{\prime}, \Gamma^{\prime}\right)=\emptyset .
$$


Let $\bar{a}=f^{-1} \varphi(a)$. Then $f(\bar{a})=\varphi(a)$. We use the condition $(*)$ for the vertex $\bar{a} \in V$. Then we see that $\Gamma-\{\bar{a}\}$ and $\Gamma^{\prime}-\{f(\bar{a})\}=\Gamma^{\prime}-\{\varphi(a)\}$ are isomorphic, i.e, there exists an isomorphism $\varphi_{0}: \Gamma-\{\bar{a}\} \rightarrow \Gamma^{\prime}-\{\varphi(a)\}$.

Let $w^{\prime}$ be a vertex in $V^{\prime}-\{f(\bar{a})\}$.

Now we show that if $\left[f(\bar{a}), w^{\prime}\right] \in E^{\prime}$ then $\left[\bar{a}, \varphi_{0}^{-1}\left(w^{\prime}\right)\right] \in E$.

Suppose that $\left[f(\bar{a}), w^{\prime}\right] \in E^{\prime}$. Then we first prove that $N\left(\varphi^{-1}\left(w^{\prime}\right)\right)-\{a\}$ is isomorphic to $N\left(\varphi_{0}^{-1}\left(w^{\prime}\right)\right)-\{\bar{a}\}$.

By $(2.1), v \notin N\left(\varphi^{-1}\left(w^{\prime}\right)\right)$. Then

$$
N\left(\varphi^{-1}\left(w^{\prime}\right)\right)-\{a\} \cong N\left(\varphi^{-1}\left(w^{\prime}\right)\right)-\{a, v\} .
$$

Since $\varphi$ is an isomorphism of $\Gamma-\{v\}$ and $\Gamma^{\prime}-\left\{v^{\prime}\right\}$, we obtain that

$$
N\left(\varphi^{-1}\left(w^{\prime}\right)\right)-\{a, v\} \cong \varphi\left(N\left(\varphi^{-1}\left(w^{\prime}\right)\right)-\{a, v\}\right) \cong N\left(w^{\prime}\right)-\left\{\varphi(a), v^{\prime}\right\} .
$$

By $(2.2), v^{\prime} \notin N\left(w^{\prime}\right)$. Hence

$$
N\left(w^{\prime}\right)-\left\{\varphi(a), v^{\prime}\right\} \cong N\left(w^{\prime}\right)-\{\varphi(a)\},
$$

i.e.,

$$
N\left(w^{\prime}\right)-\left\{\varphi(a), v^{\prime}\right\} \cong N\left(w^{\prime}\right)-\{f(\bar{a})\}
$$

Since $\varphi_{0}$ is an isomorphism of $\Gamma-\{\bar{a}\}$ and $\Gamma^{\prime}-\{f(\bar{a})\}$, we have that

$$
N\left(w^{\prime}\right)-\{f(\bar{a})\} \cong \varphi_{0}^{-1}\left(N\left(w^{\prime}\right)-\{f(\bar{a})\}\right) \cong N\left(\varphi_{0}^{-1}\left(w^{\prime}\right)\right)-\{\bar{a}\} .
$$

Thus, from (2.3), (2.4), (2.5) and (2.6), we obtain that

$$
N\left(\varphi^{-1}\left(w^{\prime}\right)\right)-\{a\} \cong N\left(\varphi_{0}^{-1}\left(w^{\prime}\right)\right)-\{\bar{a}\} .
$$

Suppose that $\left[f(\bar{a}), w^{\prime}\right] \in E^{\prime}$. Then $\left[\varphi(a), w^{\prime}\right] \in E^{\prime}$. Since $\varphi$ is an isomorphism of $\Gamma-\{v\}$ and $\Gamma^{\prime}-\left\{v^{\prime}\right\}$, we obtain that $\left[a, \varphi^{-1}\left(w^{\prime}\right)\right] \in E$. Then

$$
\begin{aligned}
& {\left[a, \varphi^{-1}\left(w^{\prime}\right)\right] \in E} \\
& \quad \Longleftrightarrow N\left(\varphi^{-1}\left(w^{\prime}\right)\right)-\{a\} \not N(s) \text { for any } s \in V, \text { by the condition (i) }
\end{aligned}
$$




$$
\begin{aligned}
& \Longleftrightarrow N\left(\varphi_{0}^{-1}\left(w^{\prime}\right)\right)-\{\bar{a}\} \not N(s) \text { for any } s \in V, \text { by }(2.7) \\
& \Longleftrightarrow\left[\bar{a}, \varphi_{0}^{-1}\left(w^{\prime}\right)\right] \in E, \quad \text { by the condition }(\mathrm{i})
\end{aligned}
$$

Thus, for any vertex $w^{\prime} \in V^{\prime}-\{f(\bar{a})\}$, if $\left[f(\bar{a}), w^{\prime}\right] \in E^{\prime}$ then $\left[\bar{a}, \varphi_{0}^{-1}\left(w^{\prime}\right)\right] \in E$.

Since $\operatorname{deg}(\bar{a})=\operatorname{deg}(f(\bar{a}))$ (i.e. $|V(N(\bar{a}))|=|V(N(f(\bar{a})))|$, we obtain that for any vertex $w^{\prime} \in V^{\prime}-\{f(\bar{a})\},\left[\bar{a}, \varphi_{0}^{-1}\left(w^{\prime}\right)\right] \in E$ if and only if $\left[f(\bar{a}), w^{\prime}\right] \in E^{\prime}$.

Here we define a map $\widetilde{\varphi}: V \rightarrow V^{\prime}$ by $\left.\widetilde{\varphi}\right|_{V-\{\bar{a}\}}=\varphi_{0}$ and $\widetilde{\varphi}(\bar{a})=f(\bar{a})$.

Then from the above observation, we obtain that $\widetilde{\varphi}$ induces an isomorphism of the two graphs $\Gamma$ and $\Gamma^{\prime}$.

Therefore the graph $\Gamma$ is reconstructible.

\section{Examples and Applications}

Example 3.1 Let $L$ be a 1-dimensional simplicial flag complex whose underlying space is Figure 1. In $L$, there are two kinds of points, that is, for a vertex $w \in L^{(0)}$, either $\operatorname{deg}(w)=2$ or $\operatorname{deg}(w)=4$.

For a vertex $w \in L^{(0)} \operatorname{such}$ that $\operatorname{deg}(w)=2$, we have

$$
\widetilde{H}_{i}(L k(w, L)) \cong \begin{cases}\mathbb{Z} & i=0 \\ 0 & i \neq 0\end{cases}
$$

and for any vertex $w^{\prime} \in L k(w, L)^{(0)}$,

$$
\widetilde{H}_{i}\left(L k(w, L)-\left\{w^{\prime}\right\}\right)=0 \quad i \geq 0 .
$$

For a vertex $w \in L^{(0)}$ such that $\operatorname{deg}(w)=4$, we have

$$
\widetilde{H}_{i}(L k(w, L)) \cong \begin{cases}\mathbb{Z} \oplus \mathbb{Z} \oplus \mathbb{Z} & i=0 \\ 0 & i \neq 0\end{cases}
$$

and for any vertex $w^{\prime} \in L k(w, L)^{(0)}$,

$$
\widetilde{H}_{i}\left(L k(w, L)-\left\{w^{\prime}\right\}\right) \cong \begin{cases}\mathbb{Z} \oplus \mathbb{Z} & i=0 \\ 0 & i \neq 0\end{cases}
$$


Hence we see that for any two distinct vertices $w, w^{\prime} \in L^{(0)}$,

$$
\begin{array}{r}
{\left[w, w^{\prime}\right] \in L^{(1)} \Leftrightarrow \widetilde{H}_{0}\left(L k(w, L)-\left\{w^{\prime}\right\}\right) \not \widetilde{H}_{0}(L k(s, L))} \\
\quad \text { for any vertex } s \in L^{(0)},
\end{array}
$$

i.e., $L$ satisfies the condition $\left(\mathrm{i}^{\prime}\right)$ in Corollary 1.5. Therefore if $L$ has enough vertices to satisfy the condition $\left(\mathrm{ii}^{\prime}\right)$, then the graph $\Gamma=L^{(1)}$ is reconstructible.

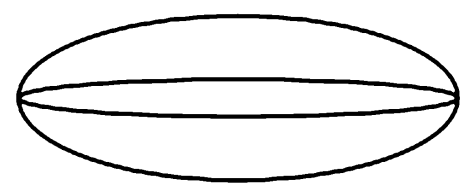

Figure 1.

Example 3.2 Let $L$ be a 2-dimensional simplicial flag complex which is a triangulation of $S^{1} *\left\{a_{1}, a_{2}, a_{3}, a_{4}\right\}$. In $L$, there are two kinds of points, that is, for a vertex $w \in L^{(0)}$, either $L k(w, L)$ is homeomorphic to $S^{1}$ or $L k(w, L)$ is homeomorphic to Figure 1 .

For a vertex $w \in L^{(0)}$ such that $L k(w, L)$ is homeomorphic to $S^{1}$, we have

$$
\widetilde{H}_{1}(L k(w, L)) \cong \mathbb{Z}
$$

and for any vertex $w^{\prime} \in L k(w, L)^{(0)}$,

$$
\widetilde{H}_{1}\left(L k(w, L)-\left\{w^{\prime}\right\}\right)=0 .
$$

For a vertex $w \in L^{(0)}$ such that $L k(w, L)$ is homeomorphic to Figure 1, we have

$$
\widetilde{H}_{1}(L k(w, L)) \cong \mathbb{Z} \oplus \mathbb{Z} \oplus \mathbb{Z},
$$

and for any vertex $w^{\prime} \in L k(w, L)^{(0)}$,

$$
\widetilde{H}_{1}\left(L k(w, L)-\left\{w^{\prime}\right\}\right) \cong 0 \text { or } \mathbb{Z} \oplus \mathbb{Z} .
$$

Hence we see that for any two distinct vertices $w, w^{\prime} \in L^{(0)}$, 


$$
\left[w, w^{\prime}\right] \in L^{(1)} \Leftrightarrow \widetilde{H}_{1}\left(L k(w, L)-\left\{w^{\prime}\right\}\right) \approx \widetilde{H}_{1}(L k(s, L))
$$

for any vertex $s \in L^{(0)}$,

i.e., $L$ satisfies the condition $\left(i^{\prime}\right)$ in Corollary 1.5. Therefore if $L$ has enough vertices to satisfy the condition $\left(\mathrm{ii}^{\prime}\right)$, then the graph $\Gamma=L^{(1)}$ is reconstructible.

Example 3.3 Let $L$ be a 3 -dimensional simplicial flag complex which is a triangulation of $S^{2} *\left\{a_{1}, a_{2}, a_{3}, a_{4}\right\}$. In $L$, there are two kinds of points, that is, for a vertex $w \in L^{(0)}$, either $L k(w, L)$ is homeomorphic to $S^{2}$ or $L k(w, L)$ is homeomorphic to $S^{1} *\left\{a_{1}, a_{2}, a_{3}, a_{4}\right\}$.

For a vertex $w \in L^{(0)}$ such that $L k(w, L)$ is homeomorphic to $S^{2}$, we have

$$
\widetilde{H}_{2}(L k(w, L)) \cong \mathbb{Z}
$$

and for any vertex $w^{\prime} \in L k(w, L)^{(0)}$,

$$
\widetilde{H}_{2}\left(L k(w, L)-\left\{w^{\prime}\right\}\right)=0
$$

For a vertex $w \in L^{(0)}$ such that $L k(w, L)$ is homeomorphic to $S^{1} *$ $\left\{a_{1}, a_{2}, a_{3}, a_{4}\right\}$, we have

$$
\widetilde{H}_{2}(L k(w, L)) \cong \mathbb{Z} \oplus \mathbb{Z} \oplus \mathbb{Z},
$$

and for any vertex $w^{\prime} \in L k(w, L)^{(0)}$,

$$
\widetilde{H}_{2}\left(L k(w, L)-\left\{w^{\prime}\right\}\right) \cong 0 \text { or } \mathbb{Z} \oplus \mathbb{Z} .
$$

Hence we see that for any two distinct vertices $w, w^{\prime} \in L^{(0)}$,

$$
\left[w, w^{\prime}\right] \in L^{(1)} \Leftrightarrow \widetilde{H}_{2}\left(L k(w, L)-\left\{w^{\prime}\right\}\right) \nsucceq \widetilde{H}_{2}(L k(s, L))
$$
for any vertex $s \in L^{(0)}$,

i.e., $L$ satisfies the condition (i') in Corollary 1.5. Therefore if $L$ has enough vertices to satisfy the condition $\left(\mathrm{ii}^{\prime}\right)$, then the graph $\Gamma=L^{(1)}$ is reconstructible. 
Here we can prove Corollary 1.7.

Proof of Corollary 1.7. In $L$, there are two kinds of points, that is, for a vertex $w \in L^{(0)}$, either $L k(w, L)$ is homeomorphic to $S^{n}$ or $L k(w, L)$ is homeomorphic to $S^{n-1} *\left\{a_{1}, a_{2}, \ldots, a_{k}\right\}$.

For a vertex $w \in L^{(0)}$ such that $L k(w, L)$ is homeomorphic to $S^{n}$, we have

$$
\widetilde{H}_{n}(L k(w, L)) \cong \mathbb{Z}
$$

and for any vertex $w^{\prime} \in L k(w, L)^{(0)}$,

$$
\widetilde{H}_{n}\left(L k(w, L)-\left\{w^{\prime}\right\}\right)=0 .
$$

For a vertex $w \in L^{(0)}$ such that $L k(w, L)$ is homeomorphic to $S^{n-1} *$ $\left\{a_{1}, a_{2}, \ldots, a_{k}\right\}$, we have

$$
\widetilde{H}_{n}(L k(w, L)) \cong \underbrace{\mathbb{Z} \oplus \mathbb{Z} \oplus \cdots \oplus \mathbb{Z}}_{k-1},
$$

and for any vertex $w^{\prime} \in L k(w, L)^{(0)}$,

$$
\widetilde{H}_{n}\left(L k(w, L)-\left\{w^{\prime}\right\}\right) \cong 0 \text { or } \underbrace{\mathbb{Z} \oplus \mathbb{Z} \oplus \cdots \oplus \mathbb{Z}}_{k-2} .
$$

Hence we see that for any two distinct vertices $w, w^{\prime} \in L^{(0)}$,

$\left[w, w^{\prime}\right] \in L^{(1)} \Leftrightarrow \widetilde{H}_{n}\left(L k(w, L)-\left\{w^{\prime}\right\}\right) \nsucceq \widetilde{H}_{n}(L k(s, L))$ for any vertex $s \in L^{(0)}$, i.e., $L$ satisfies the condition (i') in Corollary 1.5. Therefore by the Corollary 1.5 , the graph $\Gamma=L^{(1)}$ is reconstructible.

Remark The condition $k \geq 4$ in Corollary 1.7 is necessary. For example, let $k=3$ and let $L$ be a 1-dimensional simplicial flag complex whose underlying space is Figure 2. In $L$, there are two kinds of points, that is, for a vertex $w \in L^{(0)}$, either $\operatorname{deg}(w)=2$ or $\operatorname{deg}(w)=3$.

For a vertex $w \in L^{(0)}$ such that $\operatorname{deg}(w)=2$, we have 


$$
\widetilde{H}_{i}(L k(w, L)) \cong \begin{cases}\mathbb{Z} & i=0 \\ 0 & i \neq 0\end{cases}
$$

and for any vertex $w^{\prime} \in L k(w, L)^{(0)}$,

$$
\widetilde{H}_{i}\left(L k(w, L)-\left\{w^{\prime}\right\}\right)=0 \quad i \geq 0 .
$$

For a vertex $w \in L^{(0)}$ such that $\operatorname{deg}(w)=3$, we have

$$
\widetilde{H}_{i}(L k(w, L)) \cong \begin{cases}\mathbb{Z} \oplus \mathbb{Z} & i=0 \\ 0 & i \neq 0\end{cases}
$$

and for any vertex $w^{\prime} \in L k(w, L)^{(0)}$,

$$
\widetilde{H}_{i}\left(L k(w, L)-\left\{w^{\prime}\right\}\right) \cong \begin{cases}\mathbb{Z} & i=0 \\ 0 & i \neq 0\end{cases}
$$

Hence we note that $\widetilde{H}_{0}(L k(w, L)) \cong \mathbb{Z}$ for vertex $w \in L^{(0)}$ with $\operatorname{deg}(w)=2$ and $\widetilde{H}_{0}\left(L k(w, L)-\left\{w^{\prime}\right\}\right) \cong \mathbb{Z}$ for vertex $w \in L^{(0)}$ with $\operatorname{deg}(w)=3$ and vertex $w^{\prime} \in L k(w, L)^{(0)}$. Therefore $L$ does not satisfy the condition $\left(\mathrm{i}^{\prime}\right)$ in Corollary 1.5.

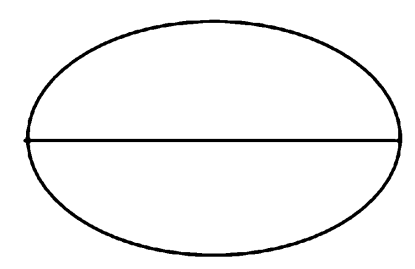

Figure 2.

Example 3.4 Here we give an example of a graph which satisfies the conditions in Theorem 1.2 but does not satisfy the conditions in Corollary 1.5 .

Let $L$ be a 2-dimensional simplicial flag complex whose underlying space is Figure 3, i.e., the underlying space of $L$ is the join of the circle and the three points set. In $L$, there are two kinds of points, that is, for a vertex $w \in L^{(0)}$, either $L k(w, L)$ is homeomorphic to $S^{1}$ or $L k(w, L)$ is 
homeomorphic to Figure 2.

We claim that $L$ does not satisfy the condition (i') in Corollary 1.5.

Indeed, for a vertex $w \in L^{(0)}$ such that $L k(w, L)$ is homeomorphic to $S^{1}$, we have

$$
\widetilde{H}_{i}(L k(w, L)) \cong \begin{cases}\mathbb{Z} & i=1 \\ 0 & i \neq 1\end{cases}
$$

and for any vertex $w^{\prime} \in L k(w, L)^{(0)}$,

$$
\widetilde{H}_{i}\left(L k(w, L)-\left\{w^{\prime}\right\}\right)=0 \quad i \geq 0 .
$$

For a vertex $w \in L^{(0)}$ such that $L k(w, L)$ is homeomorphic to Figure 2, we have

$$
\widetilde{H}_{i}(L k(w, L)) \cong \begin{cases}\mathbb{Z} \oplus \mathbb{Z} & i=1 \\ 0 & i \neq 1\end{cases}
$$

and for any vertex $w^{\prime} \in L k(w, L)^{(0)}$,

$$
\widetilde{H}_{i}\left(L k(w, L)-\left\{w^{\prime}\right\}\right) \cong \begin{cases}\mathbb{Z} & i=1 \\ 0 & i \neq 1\end{cases}
$$

or

$$
\widetilde{H}_{i}\left(L k(w, L)-\left\{w^{\prime}\right\}\right)=0 \quad i \geq 0 .
$$

Hence we note that $\widetilde{H}_{1}(L k(w, L)) \cong \mathbb{Z}$ for vertex $w \in L^{(0)}$ such that $L k(w, L)$ is homeomorphic to $S^{1}$ and $\widetilde{H}_{1}\left(L k(w, L)-\left\{w^{\prime}\right\}\right) \cong \mathbb{Z}$ for vertex $w \in L^{(0)}$ such that $L k(w, L)$ is homeomorphic to Figure 2 and vertex $w^{\prime} \in L k(w, L)^{(0)}$. Therefore $L$ does not satisfy the condition (i') in Corollary 1.5.

There exist some triangulations such that it can not apply Corollary 1.5 but it can apply Theorem 1.2.

For example, we consider a triangulation of $L$ as for a vertex $s \in L^{(0)}$ such that $L k(s, L)$ is homeomorphic to $S^{1}, L k(s, L)$ has a triangulation as Figure 4 . 


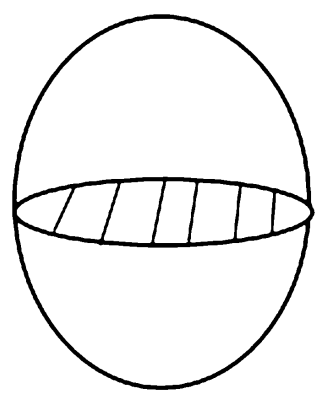

Figure 3.

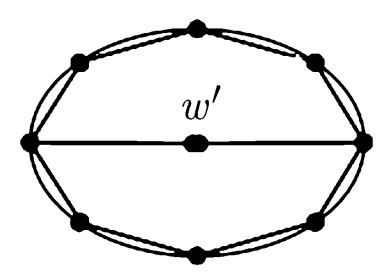

Figure 5 .

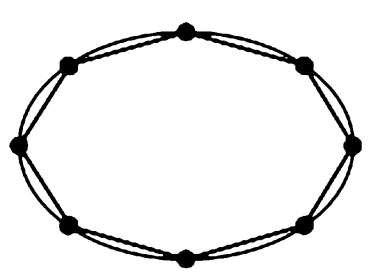

Figure 4.

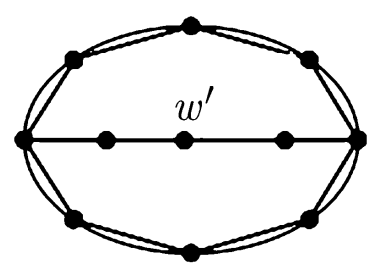

Figure 6.

(I) For a vertex $w \in L^{(0)}$ such that $L k(w, L)$ is homeomorphic to Figure 2, $L k(w, L)$ has a triangulation as Figure 5 .

In the case (I), the condition (i) in Theorem 1.2 does not hold and we cannot know whether or not the graph $\Gamma=L^{(1)}$ is reconstructible from Theorem 1.2 .

(II) For any vertex $w \in L^{(0)}$ such that $L k(w, L)$ is homeomorphic to Figure $2, L k(w, L)$ has a triangulation as Figure 6.

In the case (II), the condition (i) in Theorem 1.2 holds. Hence if $L$ has enough vertices to satisfy the condition (ii), by Theorem 1.2, the graph $\Gamma=L^{(1)}$ is reconstructible.

Acknowledgement The authors would like to express their thanks to the referees for their valuable suggestions and comments. 


\title{
References
}

[ 1 ] Bondy J. A. and Murty U. S. R., Graph Theory, Academic Press, Inc., New York, 2008.

[2 ] Bondy J. A. and Hemminger R. L., Graph reconstruction-a survey, J. Graph Theory, 1 (1977), 227-268.

[ 3 ] Davis M. W., The cohomology of a Coxeter group with group ring coefficients, Duke Math. J., 91 (1998), 297-314.

[ 4 ] Davis M. W., Poincaré duality groups, Surveys on Surgery Theory, Vol. 1 (dedicated to C. T. C. Wall, edited by S. Cappell, A. Ranicki and J. Rosenberg), pp. 167-193, Princeton Univ. Press, 1999.

[ 5 ] Hosaka T., Reconstructible graphs, simplicial flag complexes of homology manifolds and associated right-angled Coxeter groups, Osaka J. Math., to appear.

[6] Kelly P. J., A congruence theorem for trees, Pacific J. Math., 7 (1957), 961-968.

[ 7 ] Manvel B., On reconstruction of graphs, Ph.D. thesis, University of Michigan, 1970.

[ 8 ] McKay B. D., Small graphs are reconstructible, Australas. J. Combin., 15 (1997), 123-126.

[ 9 ] Mulla F. S., A class of graphs for which the Ulam conjecture holds, Discrete Math., 22 (1978), 197-198.

[10] Munkres J. R., Elements of algebraic topology, The advanced book program, Perseus Books, 1984.

[11] Quinn F., Problems on homology manifolds, Geom. Topol. Monogr., 9 (2006), 87-103.

\author{
Tetsuya Hosaka \\ Department of Mathematics \\ Shizuoka University \\ Suruga-ku, Shizuoka 422-8529, Japan \\ E-mail: sthosak@ipc.shizuoka.ac.jp \\ Yonghuo XIAO \\ Graduate School of Science and Technology \\ Shizuoka University \\ Hamamatsu, Shizuoka 432-8561, Japan \\ E-mail: f5844015@ipc.shizuoka.ac.jp
}

